\title{
BEYOND ATI: STRONG FIELD QUANTUM CONTROL
}

\author{
P. H. Bucksbaum \\ Department of Physics, University of Michigan, Ann Arbor, MI 48109-1120
}

\begin{abstract}
Above-threshold ionization (ATI) is the ionization of atoms by more than the minimum number photons necessary to overcome the Coulomb binding energy. Although ATI is contrary to the predictions of minimum-order perturbation theory, it is the dominant mode of ionization whenever the light field becomes comparable to the static fields in the atom. We have learned a great deal by studying ATI over the past several years. We now know that by controlling these high intensity optical fields, we can control atomic and molecular processes such as ionization and dissociation. We can even engineer wave-functions.
\end{abstract}

\section{INTRODUCTION}

This paper reviews the current state and future directions of super-intense laser-atom physics (SILAP). The investigations of above-threshold ionization (ATI) and high-harmonic generation (HHG) in the past few years have led to a view of the SILAP interaction in which wdvepacket dynamics play a dominant role $(1,2)$. In a companion article in this volume, $\mathrm{P}$. Corkum describes a successful model which explains many of thi: general features of ATI and HHG as the result of the scattering of an electron wavepacket by the atomic core, and the subsequent evolution of the wavepacket in the presence of the oscillating laser field (3).

Wavepacket scattering models such has those put forward and developed by Corkum, Schafer, Kulander, and others, have existed in one form or another since the first ATI experiments were performed $(4,5)$ and even before $(6-8)$. These are not the only way to view SILAP interactions; other models and techniques, such as dressed state analyses, have been similarly valuable. But these wavepacket models still represents a real breakthrough in the field, because they enable us to go beyond phenomenological investigations. Using these theories as guides, we can employ strong laser fields to probe atomic structure, to alter quantum dynamics, and even to construc. new quanturn structures. These are new and important directions in the science. 


\section{THE ATI SPECTRUM}

The ATI spectrum of xenon photoionized by $1.06 \mu \mathrm{m}$ radiation is shown in figure 1. Superimposed on this spectrum is a diagram showing how wavepacket production and scattering may produce the main features of ATI. Our description will be brief; for details, see the companion article by Corkum (3), and references therein.

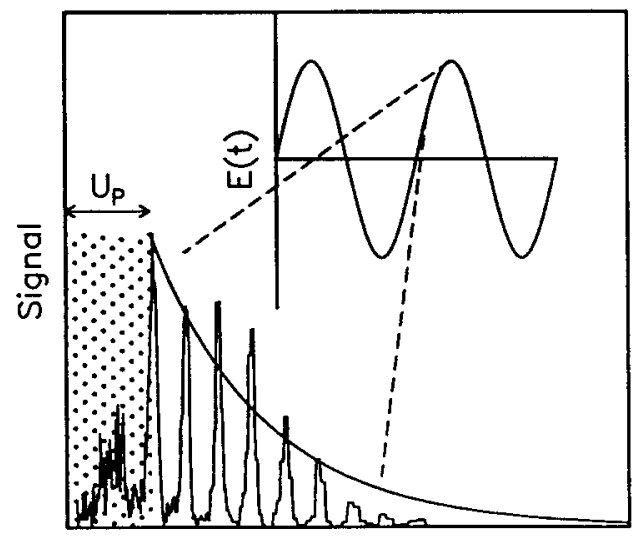

Electron energy

Figure 1. ATI spectrum of xenon ionized by $1064 \mathrm{~nm} 100$ psec laser pulses. The spectrum is shifted to higher energy by the ponderomotive energy of the beam. Its general shape follows the predictions of tunneling during each optical cycle, followed by the evolution of the free photoelectron in the optical field.

The whole spectrum of decreasing peaks is offset to higher energy by $1-2 \mathrm{eV}$. This phenomenon, which has been called "channel closure," is due to the lightinduced energy shift of the continuum relative to the ground state of the atom. Stated in terms of classical physics, the electrons produced in photoionization, even those with zero kinetic energy produced at threshold, are wiggling in the laser field. The wiggle energy, known as ponderomotive energy, is given by $U_{P}=e^{2} F^{2} /\left(4 m_{e} \omega^{2}\right)$, where $F$ is the peak amplitude of the laser field, $m_{e}$ is the electron mass, and $\omega$ is the laser angular frequency. As photoelectrons leave the laser focus on the way to detection, their wiggle energy is converted to translational kinetic energy. The process is conservative if the laser intensity is constant during the $-3-5$ psec traversal of the focus. If the wiggle energy is the principal source of AC Stark shift in the intense laser field, then this adiabatic transformation of wiggle energy to translational kinetic energy means that electrons are observed with energies that differ from the ground state energy only by the absorption of an integer number of photons. In other words, one observes multiple peaks in the spectrum which obey a generalization of Einstein's photoemission formula: $E=E_{\text {ion }}-E_{\text {accm }}+n \hbar \omega_{\text {laser }}$. 
Beyond ponderomotive effects, the essential feature of strong field ionization is that the ionization per optical cycle is no longer negligible. Therefore, the overall shape of the spectrum is controlled by variations in the tunnel-ionization rate during each optical cycle. The instantaneous ionization rate $\Gamma(t)$ for an atom with binding energy $E_{0}$ is fairly well described by the "ADK" formula (9),

$$
\Gamma(t)=4 E_{0}^{5 / 2} \frac{1}{F(t)} \exp \left[-\frac{2}{3} E_{0}^{3 / 2} \frac{1}{F(t)}\right] \text { (in atomic units), }
$$

which is an extension of the WKB tunneling rate (10) to the case of monochromatic light (11). Once ionized, the energy of the electrons evolves under the influence of the periodic driving force of the light.

Most of the features of the ATI spectrum can be derived from classical mechanics. For example, the peak ionization rate should occur at the peak of the electromagnetic field cycle. Electrons emitted by tunneling at this time enter the field with very small initial kinetic energy, and begin to wiggle in the field. Over the course of one cycle, they accelerate away from the ion, decelerate as the electric force switches direction after $1 / 4$ cycles, accelerate back towards the ion in the third $1 / 4$ cycle, and finally return to rest at their starting point one full cycle after they were ionized. If the ion Coulomb field is neglected, it is clear that these electrons just wiggle in place, with little or no drift momentum. Ponderomotive gradients in the laser focus will eventually (3-10 psec) allow the electrons to drift out of the focus, with translational energy only equal to the ponderomotive potential energy they had when created.

Electrons that tunnel out of the atom slightly before the peak of an electric field cycle experience a similar evolution, except that they have a slightly longer initial acceleration. This they keep, as drift momentum. Therefore they emerge from the focus with larger kinetic energy. There are fewer of them, because the tunneling rate when they were created is smaller. In a monochromatic laser field, the exponential increase of the tunneling rate with field strength leads to an exponential decrease of the photoelectron spectrum. This general trend is modulated into an envelope of sharp ATI peaks because of the coherent interference of ionization on successive cycles of the field.

This simple classical picture can predict most of the features of ATI experiments $(11,1)$. Furthermore, simple extensions of this picture have been used successfully to explain other high field phenomena such as high harmonic generation (12) and some aspects of multiple ionization $(13,14)$. This is truly remarkable, since the picture suffers from some obvious deficiencies: it relies on tunneling, although most ATI phenomena are observed in the intensity regime where the ionization rate per optical cycle is much less than 1 ; it ignores internal structure in the atom; and it treats the electron as a classical point particle. Nevertheless, full quantum dynamics calculations confirm that many features of SILAP interactions are essentially classical (15). 


\subsection{Intermediate Resonances}

Although the tunneling picture explains many of the phenomena of ATI, it cannot be correct on several points: the ionization rate is not given by the simple $\mathrm{ADK}$ model; in fact, the ionization is not even purely monotonic with light intensity, but is dominated by many sharp resonances (16). This was first observed in 1987, when ATI experiments were first obtained with very short pulses (17). If the pulses are shorter than about $3 \mathrm{psec}$, the ponderomotive acceleration of the free electrons is not complete. Basically, the light turns off before the electrons get out of the focus. For pulses shorter than 1 psec, new sharp peaks occur in the photoelectron spectrum. These are due to intensity-dependent multiphoton resonances between the ground state and excited states in the atom. The resonances occur in all atoms; they are caused by the huge AC stark shifts of the excited states relative to the ground state. To a good approximation, the ground state stark shift is negligible compared to the excited states, and the excited states shift by an amount equal to the ponderomotive potential shift of the continuum (18). At intensities where ATI occurs, the shift is generally on the order of one photon energy, which means that all excited states have shifted into resonance at some intensity or other during the pulse.

\subsection{Dressed State Picture}

It is easier to visualize the effect of these resonances in a dressed state picture, where they appear as avoided crossirgs between the ground state and excited states as the intensity passes through resonance. The probability of a transition during this passage depends on the size of the avoided crossing; the coupling to the continuum, which controls the ionization probability during the crossing; and the rate of crossing, which is proportional to the rate change of intensity during the pulse.

De Boer and Muller (19) pointed out that the avoided crossing leads to two different pathways to produce a photoelectron with the same energy. (a) The atom may ionize from the ground state at the intensity corresponding to a multiphoton resonance, which is basically the mechanism first proposed by Freeman et al. (17); or else (b) population may be transferred to the excited state during the crossing, and then ionization out of the excited state may occur later in the pulse, when the intensity is higher. Gibson et al. $(20,21)$ have found that the probability of ionization through ore or the other of these mechanisms can be controlled in various ways.

Recently, Story et al. (22) demonstrated this ability to control multiphoton excitation during ATI using either pulse length or the intensity where resonance occurs as control parameters. The experiment is summarized in figure 2, which shows the dressed state eigenvalues $\mathrm{ir}_{2}$ the potassium atom as a function of time during the laser pulse. They excited potassium with laser pulses of 0.5 to $13 \mathrm{psec}$ duration, and laser frequencies of $17,180 \mathrm{~cm}^{-1}$ or $17,300 \mathrm{~cm}^{-1}$. With the lower frequency light, the atom could be intensity-shifted into resonance with the $14 \mathrm{~d}$ 
state and higher, but the higher frequency light could only lead to resonances with $17 \mathrm{~d}$ and higher. The residual population in these high Rydberg states was found to depend on the rate of passage through resonance, as expected.

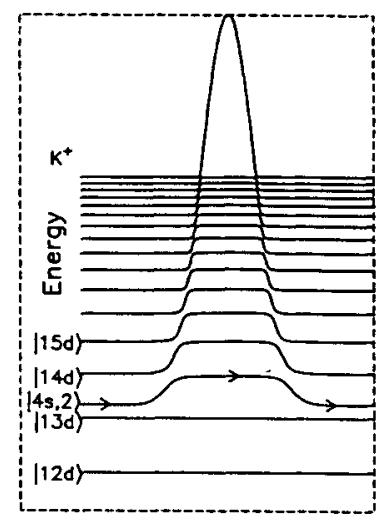

Time

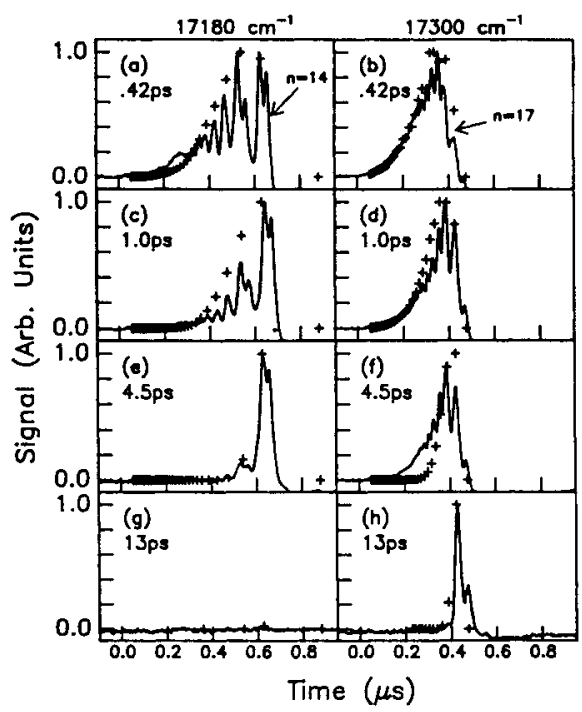

Figure 2. Dressed-state eigenvalues for 2-photon resonant, 3-photon ionization of potassium. Rydberg states are ac-Stark shifted into 2-photon resonance as the laser pulse turns on and off. The rate of intensity increase or decrease controls the probability for trapping population in the intermediate Rydberg states. (From reference 22; reprinted with permission from author.)

Passage through an avoided crossing is generally neither diabatic nor adiabatic, but rather leads to a superposition of the ground and excited states. Since the atom must pass through resonance twice, during the rising and falling edge of the laser pulse, the two passages may interfere either constructively or destructively, leading to coherent control over the left over population in the excited state after the pulse. In order to exploit this, however, it is necessary to isolate regions of the focused laser pulse where the intensity is fairly uniform; otherwise, averaging over regions with different intensity histories will wash out any coherent effects.

The experimental difficulties in observing the coherence between the two avoided crossings in the laser pulse have been recently overcome by Jones at the University of Virginia. In work reported at this ICAP meeting, Jones shows that population in sodium Rydberg states excited via multiphoton absorption can be enhanced or decreased, depending on the phase of the wave function at the two crossings.

In further work on this subject, Jones has exploited this phase control to enhance the ionization probability. The scheme works like this: if two pulses are 
incident on the atom with just the right time delay, the population transferred from the ground to the excited state on each crossing can coherently add. This can lead to an ionization enhancement for two pulses as compared to one pulse of nearly a factor of 20 ! This has been observed by Jones.

\section{ATOMIC STABILIZATION}

Strong field coherent control can also be employed to suppress the ionization rate. This "stabilization" of the atom in an intense field has been an area of active research over the past two years. Much work has concentrated on an idea proposed by Gavrila and Pont several years ago $(23,24$, and see ICAP ' 92 proceedings for details and further references). Briefly, they showed that in a very intense and very high frequency laser field, bound states become distorted along the laser polarization axis. Eventually, the electron wavefunction becomes delocalized from the atomic potential. This reduces the binding energy of the state, and also reduces the ionization cross section.

The high frequency approximation used by Gavrila and Pont states that $\hbar \omega \gg E_{0}$. This is not realized for ground state atoms in optical laser fields; however, since the distortion of the wave function eventually reduces the binding energy of all bound states, they conclude that even tightly bound states eventually reach the high frequency stabilization regime. The lifetime of the atom in a laser field can be plotted vs the laser intensity. There are three distinct regions. In the lowest intensity region, the one of ordinary ATI, the lifetime decreases steeply with intensity. As the intensity rises, eventually the atom reaches the tunneling regime where the lifetime is comparable to a single optical cycle. This is the second region, which has become known as "Death Valley." Beyond this is the third region, the regime of high frequency stabilization. The experimental challenge has been to devise a way for an atom to survive Death Valley as the laser intensity rises on the leading edge of the laser pulse.

Recently, de Boer and coworkers have found evidence for high frequency stabilization (25). They eryoloyed several experimental tricks to achieve the stabilization regime. First, th:ey worked with atoms in highly excited states, so that the high frequency approximation is valid throughout the experiment. Their most important innovation was to prepare states with high 1 and m quantum numbers, so-called "circular states." In these states, Death Valley is considerably shallower, thus insuring that some population will survive the rising edge of a $100 \mathrm{fsec}$ laser pulse.

The experiment was performed on $5 \mathrm{~g}$ states in neon (25). Figure 3 is a schematic of the experimental arrangement. 5-photon excitation from the ground state with circularly polarized $1 \mathrm{psec} 286 \mathrm{~nm}$ pulses prepares neon in the $5 \mathrm{~g} \mathrm{~m}=4$ state. Actually, the experiment utilized adiabatic transfer of population at an intensity-induced avoided crossing between the ground state and the $5 \mathrm{~g}$ state, just as in the experiment of Story et al. described above. The crossing occurs at $\mathrm{I}=8.6 \times 10^{13} \mathrm{~W} / \mathrm{cm}^{2}$. Ti.e ionization of the $5 \mathrm{~g}$ state saturates at $4 \times 10^{14} \mathrm{~W} / \mathrm{cm}^{2}$, 
so it is possible to excite a significant fraction of the ground state without ionizing.
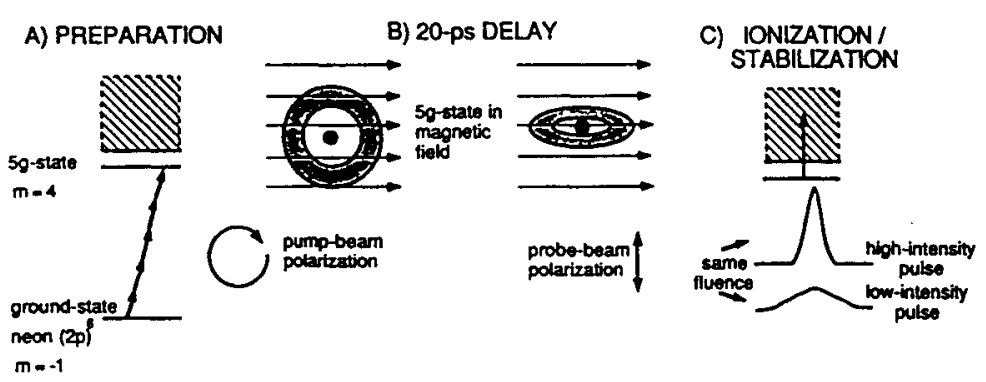

Figure 3. The experimental scheme emplcyed in reference 25. Multiphoton excitation of the $5 \mathrm{~g}$ circular state in $\mathrm{Ne}$ is followed by irradiation by an intense pulse. Leftover population is monitored to find evidence of stabilization. (Reprinted with permission from author.)

For circular states, Gavrila's stabilization theory requires a laser polarization along $\hat{z}$, that is, normal to the plane of the orbit. To accomplish this, the researchers allowed the atom to precess $\pi / 2$ radians in a $0.9 \mathrm{~T}$ magnetic field. Then they illuminated it with a $620 \mathrm{~nm}$ probe pulse of various duration and peak intensity, and observed the ionization of the $5 \mathrm{~g}$ state. Figure 4 shows the resulting spectra. The 5g peak appears to saturate if the pulses are intense enough. The results are undramatic, but significant. This is the first evidence that high frequency adiabatic stabilization occurs.

\section{MOLECULAR STABILIZATION}

There has also beeri considerable effort to control molecular processes with short lasers. In $\mathrm{H}_{2}^{+}$, the the AC Stark effect creates gaps in the dressed state internuclear potentials for the ground state and first excited state (see figure 5). These are quite analogous to the dressed-state avoided crossings in atoms (see figure 2 above). Population may transfer from one state to the other at these gaps during an intense laser pulse, and control may be possible.

One of the more interesting aspects of these laser-induced avoided crossings is the presence of new light-induced vibrational potential wells formed above them. Recent work by Mies and Giusti suggest that population bound in the vibrational states of $\mathrm{H}_{2}^{+}$will be partly trapped in these new vibrational states if the laser pulse is sufficiently short (26). Zavriyev et al. (27) has obtained experimental evidence for this light-induced structure in recent experiments.

\section{CONTROL STRATEGIES}

The experiments des:ribed above all achieve some degree of control over the ionization process by adj'sting the duration, wavelength, and intensity of a nearly 

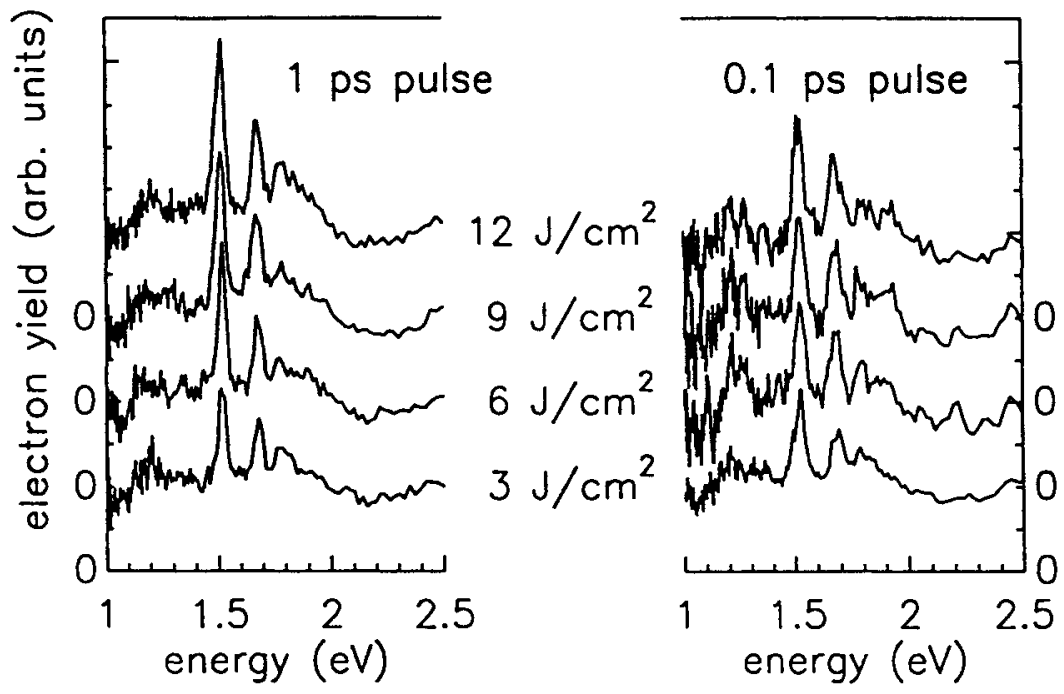

Figure 4. Photoelectron spectra showing evidence of adiabatic stabilization. For long pulses, higher fluence leads to less population left over in the $5 \mathrm{~g}$ state in neon. For short pulses, the population decrease saturates. (From reference 25; reprinted with permission)

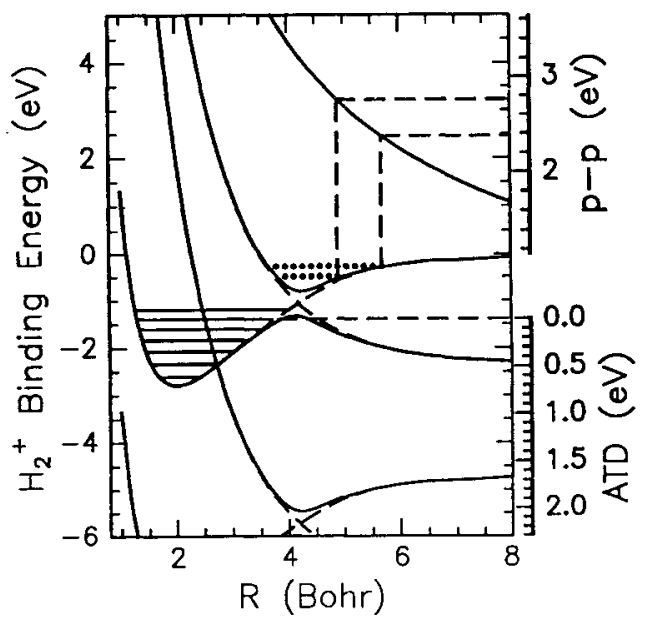

Figure 5. Laser-dressed energy levels of $\mathrm{H}_{2}^{+}$in a $3.5 \mathrm{TW} / \mathrm{cm}^{2}, 532 \mathrm{~nm}$ laser field. Energies of proton fragments fo by:ing dissociation are shown on the right side.

Gaussian laser pulse. However, current short pulse laser technology allows much more significant alteration of the form of short laser pulses. In the remainder of this paper, we will discuss several new methods for exerting more sophisticated control over atoms with strong laser fie'ds. 


\subsection{Optical Frequency Multiplexing}

One important method of increasing control over atomic photoionization and molecular dissociation is by combining two or more laser colors. In weak field experiments, this can produce multiple pathways which may interfere depending on the relative phase of the two laser fields. If the frequencies are commensurate, such a phase can always be defined $(28,29)$. In a strong field experiment, we enter the tunneling regime described in figure 1: the instantaneous tunneling rate during each field cycle depends on the magnitude of the instantaneous electric field, which is just the superposition of the various laser fields. 2-color experiments are critical tests of these theories (30).

There is no well-defined field magnitude where perturbative multiphoton ionization gives way to tunneling; however, the transition is fairly rapid in high order multiphoton processes, since the ionization rate is changing as a high power of the intensity. The Keldysh parameter $\gamma=\sqrt{\mathrm{E}_{0} / 2 \mathrm{U}_{\mathrm{P}}}$ (31) is a convenient dimensionless figure of merit for this. $\gamma$ is roughly one over the product of the tunneling rate and one-quarter of ar. optical period, which is the time available for tunneling near each half-cycle maximum of the laser field. When $\gamma<1$, ionization is expected to be dominated by tunneling. Under these conditions, the phasedependence of ionization in a 2-color field is just due the phase-dependence of the field shape: highest rates occur when the fields add constructively, and the tunnel barrier is most suppressed.

Several experiments have begun to test these ideas (32-35). Schumacher (35) recently showed that it is possible to focus an intense laser beam and its second harmonic into an atomic gas so that the phase shift between the two colors is stable, measurable, and well-controlled. He used this control to test the two-step model of above-threshold ionization outlined above.

In Schumacher's experiment, there is a two-color pulsed laser field $\xi(t)$ consisting of a field $F_{1}(t)$ with fundamental frequency $\omega$ produced by an amplified mode-locked Nd:YAG laser ( $\tau=100 \mathrm{psec}, \lambda=1064 \mathrm{~nm}$ ), and its second harmonic, $\mathrm{F}_{2}(\mathrm{t}) . \xi(\mathrm{t})$ is the coherent sum field

$$
\xi(t)=F_{1}(t) \cos \omega t+F_{2}(t) \cos (2 \omega t+\phi)
$$

The fields are equal in magnitude, and they are phase-locked to each other because $F_{2}(t)$ is produced via second harmonic generation in a $\mathrm{KD} * \mathrm{P}$ crystal. Care is taken to focus the two beams so that their relative phase in the focus is constant and stable. Their polarizations are parallel. The amplitude and shape of $\xi(t)$ depends on the relative phase $\phi$, which can be determined absolutely using nonlinear frequency mixing and optical rectification in a second $\mathrm{KD} * \mathrm{P}$ crystal placed inside the vacuum chamber where the experiment takes place.

The experiment was performed in Krypton, where at least $12(1064 \mathrm{~nm})$ photons are required to ionize. Figure 6 shows a 2-color ATI spectrum for $\phi=0$, and $F_{1}=F_{2}=1.2 \mathrm{~V} / \AA$. (Total intensity of $4 \times 10^{13} \mathrm{~W} / \mathrm{cm}^{2}$.) Electrons are detected 


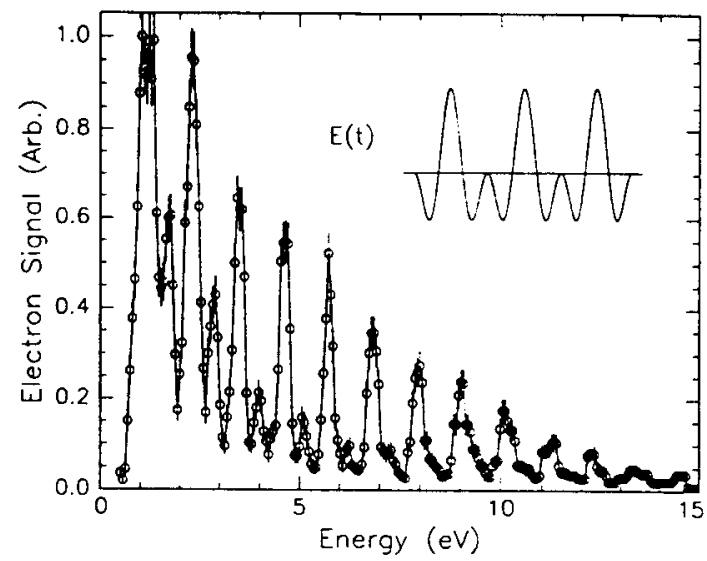

Figure 6. Krypton ATI photoelectron spectrum for equal amplitude $532 \mathrm{~nm}$ and $1064 \mathrm{~nm}$ radiation, with relative phas $=\phi=0$. (From reference 35 , reprinted with permission.)

emerging along the polarization axis, but in only one direction. (This makes it possible to observed left/right asymmetries in the ionization spectrum.) There are two series of peaks (large and small) corresponding to two different final states in the ion. The $\phi$-dependence for the third through sixth large peaks $\left({ }^{2} \mathrm{P}_{3 / 2}\right.$ final state) are shown in figure 7 together with the predictions of the 2-step model. It is quite clear that by controlling the phase, one can control the spectrum. For example, the phases with the highest ionization rates do not produce the highest energy electrons.

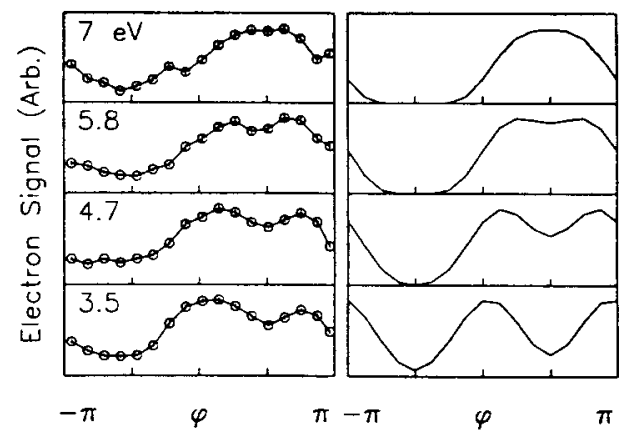

Figure 7. dependence of ATI peaks 3 through 6 , for total intensity of $4 \times 10^{13} \mathrm{~W} / \mathrm{cm}^{2}$. Tunneling model predictions shown at right. (From reference 35 , reprinted with permission.) 
As the intensity goes up, the data start to deviate from the predictions of the simple model. The two-step model describes the essential physics for low intensities and/or for low energy electrons; but high energy electrons are produced at high intensities by some additional process. Again the phase dependence is critical to this observation because the excess high energy electrons are produced predominantly in one direction. Excess high energy electrons have been seen in other experiments as well $(2,36)$. The mechanism for producing the excess is still not clear, but one plausible idea proposed by several researchers is core rescattering following ionization (2). The electrons scattered by their parent ion are reaccelerated by the laser field, and on average they gain initial kinetic energy. This is essentially the same mechanism as radio-frequency heating in plasmas, except that here it is the parent ion that scatters the electron.

Schumacher also tested this hypothesis by comparing his linear polarization results with those obtained if he introduced a small amount of elliptical polarization. The idea is that the probability of core rescattering is greatly reduced in elliptically polarized light, since the electron is diverted from the parent ion by the out-of-phase component of the field. Indeed, the large phase asymmetry seen for high intensity 2-color ATI disappeared, lending support to the rescattering argument.

\subsection{Mixing Colors in Dissociation Experiments}

2-color strong-field laser control has also been discussed as a means of controlling molecular dissociation $(37,38)$. Here the mixing of $\omega$ and $2 \omega$ breaks the left-right symmetry along the polarization axis. Recent calculations show how frequency multiplexing may be used to align molecules and to select the channel or even the directions of the fragments (39). The simplest case is again $\mathrm{H}_{2}^{+}$, where the fragments are a proton and a hydrogen atom. Here, different above-threshold dissociation channels can open and close, and the proton can be preferentially directed in either sense along the polarization direction (39). In the case of HD, Mies and Giusti suggest that isotopic selectivity may also be possible ,in the sense that the deuteron and proton may be ejected in different preferred directions (40). Experiments to test these ideas are underway at Brookhaven and Michigan.

\subsection{Pulse Shaping}

Frequency mixing is not the only way to modulate intense laser fields. A more general technique is pulse-shaping, where the laser electric field may by sculpted into a desired form by frequency and phase modulations. This is no longer a hypothetical possibility; liquid crystal modulators have now been employed to provide independent, programmable amplitude and phase control in amplified sub-picosecond pulses.

A group at the University of Michigan used amplified programmable shaped optical pulses recently to engineer Rydberg wavepackets (41). In the strong field regime, any arbitrary va':epacket superposition can be obtained by manipulating the light that creates the excited state. The demonstration experiment uses $110 \mathrm{fs}$ 
$785 \mathrm{~nm}$ pulses containing about $10 \mathrm{~nm}$ of bandwidth, obtained from a Ti:Sapphire Kerr-lens modelocked laser. The pulse shaper is built in a zero-dispersion grating-pulse expander. This is essentially two diffraction gratings with an inverting telescope inbetween. This device can be configured to produce no net dispersion; however, in the middle of the telescope where where the light is focused, all the frequency components are dispersed in the horizontal plane. Two liquid crystal displays (LCD's) similar to those found in laptop computers intersect the beam at this point (42). These produced independent computer-controlled phase delays or polarization shifts, providing both phase and amplitude control over each color in the pulse. The pulses are amplified to $1 \mathrm{~mJ}$ in a standard chirped-pulse amplifier (43). The shape following amplification is characterized using a frequencyresolved optical gate (44).

The nonstationary states excited by the shaped pulses in the demonstration experiment are constructed from the np Rydberg states in Cs. Population in the Rydberg states is monitored using ramped-field ionization. In this common analysis technique, a slowly ramping dc electric field after the laser pulse ionizes the wavepacket as it reaches its field-ionization threshold $\left(F=1 /\left(2 n^{*}\right)^{4}\right)$. In this way, the np Rydberg states making up the wavepacket are dispersed in time in the detector.

The simplest way to see the effect of the pulse shaper is to observe spectral shaping in the Rydberg populations observed during ramped field ionization. Figure 8 shows two ramped-field spectra: one from a near-Gaussian pulse, and the second from a pulse in which frequencies corresponding to all but four $\mathrm{np}$ states have been selectively removed. Such a pulse is easy to produce in the pulse shaper.

A more sophisticated technique for observing shaped pulses is the "optical Ramsey method" as follows (45): The shaped light is split into two identical pulses with a Michelson interferometer. The time separation between the pulses can be varied over seve: ai picoseconds. Each pulse in the pair excites Cesium from a pre-excited $7 \mathrm{~s}$ state to a sculp:ed wave function or "wavepacket", i.e. a coherent superposition of $n p$ states centered around $n=27$. The two wavepackets are phase-coherent with respect to each other so there is interference between them. This changes the iotal population excited into each Rydberg state as the time delay is moved. Tre population vs. time is an interferometric autocorrelation of the complex scalar wisunction of the excited atom.

In the limit of little depletion of the $7 \mathrm{~s}$ state, this autocorrelation is related to the fourier transform of the spectral amplitude function of the pulse, much as a Michelson autocorrelation of an optical field can be used for spectroscopy. In strong fields, The depletion of the is stated by the first pulse changes the wavepacket excited by the second pulse. This additional feature might be used to extract phase information about the wavepacket as well. 


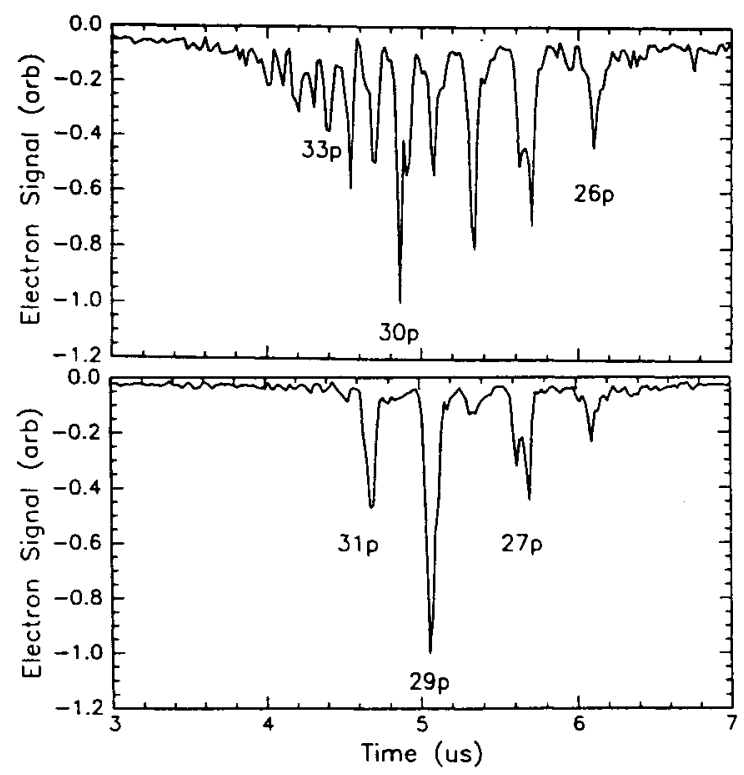

Figure 8. Top: ramped field spectrum from a Gaussian pulse. Bottom: ramped field spectrum from a shaped pulse.

Figure 9 shows the autocorrelation of the Cs wavepacket produced by the shaped pulse shown above. A calculation is also shown for comparison.

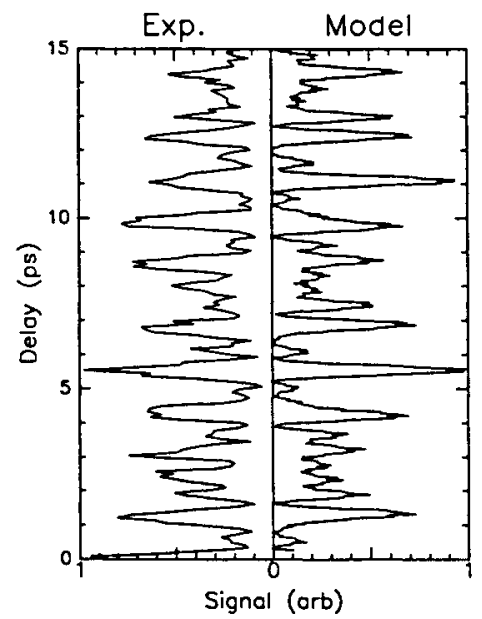

Figure 9. Autocorrelation of a 3-state cesium wavepacket produced by a shaped optical pulse. Also shown is a calculation of the expected autocorrelation function.

\subsection{Half-Cycle Pulses}

Ultimate quantum control requires the ability to sculpt any arbitrary wavefunction. This is certainly beyond reach with present technology. However, for a 
large number of problems in ionization, dissociation, or bond-selective chemistry, we can set a more modest goal of manipulating the wavefunction on a timescale comparable to the quantum dynamics. For Rydberg atoms, and for most molecules, present ultrafast laser technology ( $\left.\tau_{\text {pulse }} \geq 20 \mathrm{fsec}\right)$ is nearly sufficient, provided we have total control of the light field in this timescale. Therefore, a great emphasis is placed on the ability to expand the usable coherent bandwidth in the pulse.

In this final section, we will describe recent advances in manipulating ultrashort far infrared pulses. The number of field cycles in these pulses can actually approach the dc limit where the field merely traces out the upper or lower envelope of the optical pulse train. We call these "half-cycle pulses" (HCP), since they contain less than one full optical cycle.

Large $(-10-100 \mathrm{kV} / \mathrm{sm}) \mathrm{HCP}$ 's are produced when a $100 \mathrm{fsec}$ optical pulse is incident on a field-biased airect-gap semiconductor such as GaAs or InP $(46,47)$. The electric field in the pulse is produced by the rapidly changing photocurrent in the semiconductor following excitation, and propagates in the direction of the incident laser due to elementary phase-matching requirements. Since the HCP is freely propagating, it can be reflected from metal mirrors, focused, and directed into an atomic or molecular beam or cell. Typically, the pulse is about $400 \mathrm{fsec}$ in duration, with a coherent bandwidth of more than $1 \mathrm{THz}$.

The "half-cycle" nature implies that the time integral of the field is not zero. This is possible because the current distribution in the semiconductor is not the same before and after th $ə$ pulse, i.e. there is no current prior to excitation, but -1 psec afterwards a dc current is flowing through the semiconductor. There is a slow recharge period of several hundred $\mu \mathrm{sec}$, when the dc current turns off. This produces a tiny but persistent negative tail in the field, so that, over milliseconds, the time integral really does vanish. However, for the purpose of manipulating wavefunctions with dyramical timescales of $100 \mathrm{fsec}$ to a few psec, the field is nearly unipolar, hence "half-cycle."

Rydberg states in alkali atoms show off the unique features of HCP's. Here we will show the results of two experiments in our laboratory. In the first, Jones et al (48) studied the stability of Rydberg ns and nd states in Na to HCP's. The states are bound by $100 \mathrm{~cm}^{-1}$ or more, so that single photons within the HCP coherent bandwidth cannot ionize. the atom. Furthermore, dc field ionization is not expected, because the duration of the pulse is much shorter than the Kepler orbit time of the atom. Expressed in terms of wave mechanics, this means that most of the wavefunction does not sample the saddle-point in the potential during the pulse. The data are summarized in figure 10, and show some remarkable trends. First, the ionization vs field strength for each $\mathrm{n}$ state displays neither a power law nor a sharp ti:eshold behavior, instead, there is a slow turn-on, followed by a rapid rise to raarly $100 \%$ icnization. 


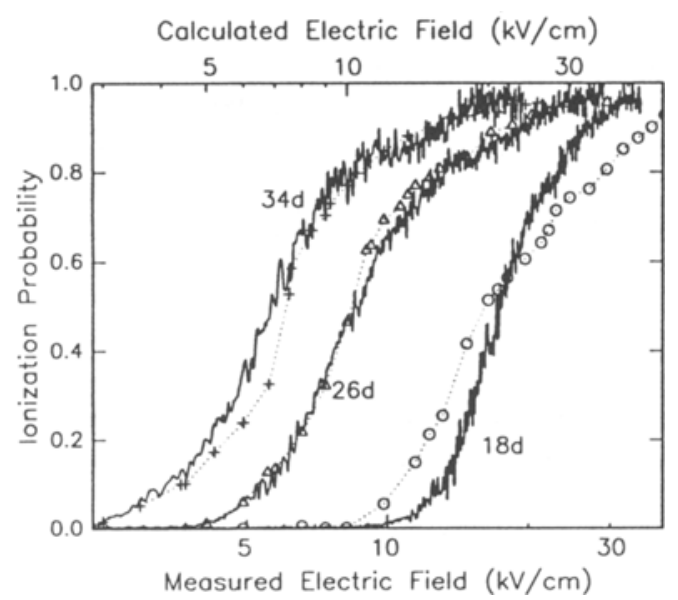

Figure 10. Ionization signal as a function of the peak electric field in the half-cycle pulse, for $18 d, 26 d, 34 d$ initial states in $\mathrm{Na}$. The smooth curves are classical simulations (48) for these states. Best agreement occurs for a rescale factor of 1.3 in the experimental electric field. (From ref. 47, reprinted with permission.)

A pulse of the same duration and bandvidth, but centered at optical frequencies, has a completely different behavior (49-51). For optical pulses, ionization can only take place near the ion core, where the electron can exchange momentum with the ion. Most of the state probability density is located at large distances from the core, so the ionization probability saturates at only a few percent ionization. This behavior has been studied extensively, and is now usually described as the formation of a trapped nonstationary state called a "dark wavepacket."

We can understand the different behavior of HCP ionization from simple classical dynamics argumerts. The key is that an HCP electric field has a nonzero time integral, so that it car: transfer much more momentum to an electron than the photon momentum hc/ $\lambda$. The electron receives a kick from the field which changes its momentum by

$$
\delta p=\int_{-\infty}^{\infty} F(t) d t,
$$

where $\mathbf{F}(t)$ is the HCP field. Even electrons at rest can receive substantial energy from this. More generally, we can integrate the energy change:

$$
\Delta E=-\int_{-\infty}^{\infty} F(t) \cdot v(t) d t
$$

where $v(t)$ is the velocity of the electron. The results of the classical simulation are shown in figure 10 . 
Perhaps the most dramatic illustration of the effect of HCP's on atoms was in a recent experiment performed at Virginia and Michigan on "oriented" Rydberg states (52). Such states are produced when an electric field is used to split the 1degeneracy of Rydberg atoms. This permits the selective excitation of states with permanent electric dipole moments, where the electron wave function is predominantly on one side of the atom. Then the ionization probability depends not only on the magnitude of the HCP, but also on its orientation relative to the atom. Atoms ionize most easily when the HCP produces a force on the electron in the direction away from the ion core. These results can be qualitatively understood classical arguments. Figure 11 shows ionization curves for two states with the opposite orientation, alor.g with classical simulations.

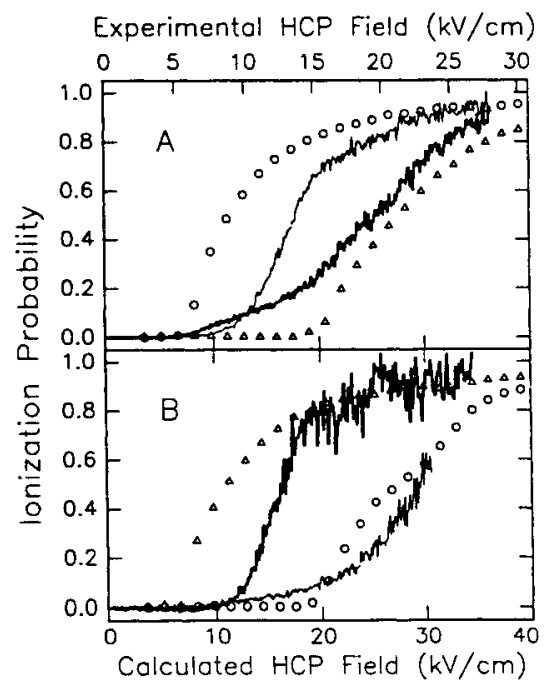

Figure 11. Ionization signial $n=17$ states in $\mathrm{Na}$. with two different orientations: (a) dipole moment along $-\hat{z}$ (b) dipc! $\in$ moment along $+\hat{z}$. Light and dark lines are for HCP field directions $-\hat{z}$ and $+\hat{z}$, respectively. $(0)$ and $(\Delta)$ trace classical simulation results for $-\hat{z}$ and $+\hat{z}$ HCP directions, respectively. From ref. 52, with permission.

\section{ACKNOWLEDGEMENTS}

We thank Robert R. Jenes, Douglass W. Schumacher, Harm G. Muller, and M. P. de Boer for valuable discussions, and we thank several authors for permission to reprint their figures in this work. This work was supported by the National Science Foundation. Part of this work was supported by a Nato Collaborative Research Grant. 


\section{REFERENCES}

1. Corkum, P.B., Physical Review Letters 71, 1994, (1993).

2. Schafer, K.J., Yang, B., DiMauro, L.F., Kulander, K.C., Physical Review Letters 70 1599-1602 (1993)

3. Corkum, P.B., in this volume.

4. P. Agostini, F. Fabre, G. Mainfray, G. Petite, and N. Rahman, Phys. Rev. Lett. 42, 1127 (1979).

5. P. Kruit, J. Kimman, H. G. Muller, and J. J. van der Wiel, Phys. Rev. A28, 248 (1983).

6. L. V. Keldysh, Sov. Phys. JETP 20, 1307 (1965).

7. F. H. M. Faisal, J. Phys. B 6, L89 (1973);

8. H. R. Reiss, Phys. Rev. A 22, 1786 (1980).

9. Ammosov, M. V., Delone, N. B., and Krainov, V. P. Sov. Phys. JETP 64, 1191 (1986).

10.See, for example, L.D. Landau and E.M. Lifshitz, Quantum Mechanics, Course of Theoretical Physics Vol. 3 (Pergamon, New York, 1965),

11. P. B. Corkum, N. H. Burnett, and F. Brunell, Phys. Rev. Lett. 62, 1259 (1989).

12. M. Lewenstein, P. Balcou, M.Y. Ivanov, A. L'Huillier, P.B. Corkum, Physical Review A 49, 2117 (1994).

13. K. Kondo, A. Sagisaka, T Tamida, Y. Nahekawa, and S. Watanabe, Phys. Rev. A 48, R2531 (1993).

14. B. Walker, E. Mevel, Baorui Yang, P. Breger, J.P. Chambaret, A. Antonetti, L.F. DiMauro, P. Agostini, Physical Review A 48, R894 (1993).

15. K. C. Kulander, K. J. Schafer, J. L. Krause, Laser Physics 3, 359 (1993).

16. P. H. Bucksbaum, L. Van Woerkom, R. R. Freeman, and D. W. Schumacher, Phys. Rev. A 41, 4119 (1990).

17. R.R. Freeman, P.H. Bucksbaium, H. Milchberg, S. Darack, D. Schumacher, and M.E. Geusic, Phys. Rev. Letters 59, 1092 (1987).

18. L. Pan, L. Armstrong, Jr., and J. H. Eberly, J. Opt. Soc. Am. B 3, 1319, (1986).

19. M. P. de Boer and H. G. Muller, Phys. Rev. Lett. 68, 2747, (1992).

20. G.N. Gibson, R.R. Freeman, T.J. Mcllrath, Physical Review Letters 69, 1904 (1992).

21. G.N. Gibson, R.R. Freman, T.J. Mcllrath, and H.G. Muller, Physical Review A 49, 3870 (1994).

22. J.G. Story, D.I. Duncan, T.F. Gallagher, Physical Review Letters 70, 3012 (1993).

23. M. Pont, N.R. Walet, M. Gavrila, and C.W. McCurdy, Phys. Rev. Lett. 61, 939 (1988).

24. M. Pont, Phys. Rev. A 40, 5659 (1989).

25. M. P. de Boer, J. H. Hoogenraad, R. B. Vrijen, L. D. Noordam, H. G. Muller, Physical Review Letters 71, 3263 (199:).

26. A. Giusti-Suzor, F.H. Mies, Physical Review Letters 68, 3869 (1992).

27. A. Zavriyev, P.H. Bucksbaum, J.Squier, and F. Saline, Physical Review Letters 70, 1077 (1993).

28. Yi-Yian Yin, Ce Chen, and D.S. Elliot, Phys. Rev. Lett., 69, 2353 (1992).

29. Ce Chen, Yi-Yian Yin, and D.S. Elliot, Phys. Rev. Lett, 64, 507 (1990).

30. K. J. Schafer and K. C. Kulander, Phys. Rev. A., 45, 8026 (1992).

31. L.V. Keldysh, Sov. Phys. IETP, 20, 1307 (1965).

32. H.G. Muller, P.H. Bucksbaym, D.W. Schumacher, and A. Zavriyev, J. Phys. B, 23, 2761, (1990)

33. D. Normand, L.A. Lompre, A. L'Huillier, J. Morellec, M. Ferray, J. Lanvancier, G. Mainfray, and C Manus, J. Opt. Soc. Am. B 6, 1513 (1989)

34. K. Kondo, Y. Nabekawa, and S. Watanabe, in abstracts of High field interactions and short Wavelength Generation, St. Malo, August 1994.

35. D.W. Schumacher, F. Weihe, H. G. Muller, and P. H. Bucksbaum, Phys. Rev. Lett. 73, 1344 (1994).

36. U. Mohideen, M. H. Sher, H. W. K. Tom, G. D. Aumiller, O. R. Wood, R. R. Freeman, J. Bokor, and P. H. Bucksbaum, Phys. Rev. Let. 71, 509 (1993). 
37. E. Charron, A. Giusti-Suzor, F. H. Mies, Physical Review Letters 71, 692 (1993).

38. A. D. Bandrauk, J.-M. Gauthier, J. F. McCann, Journal of Chemical Physics 100, 340 (1994).

39. E. Charron, A. Giusti-Suzor, F. H. Mies, Physical Review A 49, R641 (1994).

40. E. Charron, A. Giusti-Suzor, F. H. Mies, submitted.

41. P. H. Bucksbaum, D. W. Schumacher, J. H. Hoogenraad, Jeffrey L. Krause, Kent R. Wilson, Proceedings of Ultrafast '94, ed. by P. Barbara and W. Knox, Springer Verlag, (1994).

42. A. M. Weiner, D. E. Leaird, J.S. Patel, and J. R. Wullert II, IEEE JQE 28, 908 (1992).

43. J. Squire, F. Salin, G. Mourou, and D. Harter, Op. Lett. 16, 324 (1991).

44. D. J. Kane, R. Trebino, Opt. Lett. 18823 (1993).

45. R. R. Jones, C. S. Raman, D. W. Schumacher, and P. H. Bucksbaum, Physical Review Letters 71, 2575 (1993).

46. D. You, R. R. Jones, D.R. Dykaar, and P.H. Bucksbaum, Optics Letters 18, 290 (1993).

47. R. R. Jones and P. H. Bucksbaum, Comments on Atomic and Molecular Physics, in press (1994).

48. R.R. Jones, D. You, and P.H. Bucksbaum, Physical Review Letters 70, 1236 (1993).

49. R.R. Jones and P.H. Bucksbaum, Phys. Rev. Lett. 67, 3215 (1991)

50. H. Stapelfeldt, D.G. Papaioannou, L.D. Noordam, and T.F. Gallagher, Phys. Rev. Lett. 67, 3223 (1991).

51. R.R. Jones, D.W. Schumacher, and P.H. Bucksbaum, Phys. Rev. A 47, R49 (1993).

52. R.R. Jones, N.E. Tielking, D. You, C. Raman, and P.H. Bucksbaum, submitted. 